\title{
Elucidating tumor heterogeneity from spatially resolved transcriptomics data by multi-view graph collaborative learning
}

\section{Chunman Zuo}

Institute of Artificial Intelligence, Donghua University

\section{Yijian Zhang}

Xinhua Hospital affiliated to Shanghai Jiaotong University School of Medicine

\section{Chen Cao}

University of Calgary

Jinwang Feng

School of Automation, Northwestern Polytechnical University

Mingqi Jiao

Hangzhou Institute for Advanced Study

Luonan Chen ( $D$ Inchen@sibs.ac.cn )

Chinese Academy of Sciences

\section{Article}

Keywords:

Posted Date: February 10th, 2022

DOI: https://doi.org/10.21203/rs.3.rs-1287670/v1

License: (c) (1) This work is licensed under a Creative Commons Attribution 4.0 International License.

Read Full License

Version of Record: A version of this preprint was published at Nature Communications on October 10th, 2022. See the published version at https://doi.org/10.1038/s41467-022-33619-9. 
Elucidating tumor heterogeneity from spatially resolved transcriptomics data by multiview graph collaborative learning

Chunman Zuo ${ }^{1, *}$, Yijian Zhang ${ }^{2}$, Chen $\mathrm{Cao}^{1}$, Jinwang Feng ${ }^{3}$, Mingqi Jiao ${ }^{4}$, and Luonan Chen $^{4,5,6,7, *}$

${ }^{1}$ Institute of Artificial Intelligence, Donghua University, Shanghai 201620, China

${ }^{2}$ Department of General Surgery, Xinhua Hospital Affiliated to Shanghai Jiao Tong University School of Medicine, Shanghai 200092, China

${ }^{3}$ Key Laboratory of Information Fusion Technology of Ministry of Education, School of Automation, Northwestern Polytechnical University, Xi'an 710072, China

${ }^{4}$ Key Laboratory of Systems Health Science of Zhejiang Province, Hangzhou Institute for Advanced Study, University of Chinese Academy of Sciences, Chinese Academy of Sciences, Hangzhou 310024, China

${ }^{5}$ Key Laboratory of Systems Biology, Shanghai Institute of Biochemistry and Cell Biology, Center for Excellence in Molecular Cell Science, Chinese Academy of Sciences, Shanghai 200031, China

${ }^{6}$ Guangdong Institute of Intelligence Science and Technology, Hengqin, Zhuhai, Guangdong 519031, China

${ }^{7}$ School of Life Science and Technology, ShanghaiTech University, Shanghai 201210, China

* To whom correspondence should be addressed.

Email: cmzuo@dhu.edu.cn; lnchen@sibs.ac.cn 


\begin{abstract}
Spatially resolved transcriptomics (SRT) technology enables us to gain novel insights into tissue architecture and cell development, especially tumors. However, the lack of effective methods for exploiting biological contexts (e.g., global position information) and multi-view features has severely hindered the disentangling ability for tissue heterogeneity. Here, we proposed stMVC, a multi-view graph collaborative learning model that integrates histology, gene expression, spatial location, and biological contexts in analyzing SRT data by attention. Specifically, stMVC adopting semi-supervised graph attention autoencoder separately learns view-specific representations for each of two graphs, i.e., histological similarity graph by visual features and spatial location graph by physical coordinates, and then simultaneously integrates two-view graphs for robust representations via learning weights of different views with attention in a semi-supervision manner from biological contexts. Benchmark studies of stMVC on 12 slices from the human cortex, demonstrate its superior capability in detecting tissue structure, visualizing trajectory relationships between different layers, and denoising data. In particular, in the breast cancer study, stMVC identified new disease-related cell-states and their transition cell-states, which were further validated by the functional and survival analysis of independent clinical data. Those results not only provided novel biological insights into tumor heterogeneity but also demonstrated clinical and prognostic applications from SRT data. The software is available at https://github.com/cmzuo11/stMVC.
\end{abstract}

\title{
Introduction
}

The recent technological innovation of SRT platform allows profiling gene expression patterns in the spatial contexts of tissue ${ }^{1}$, such as Visium from $10 \mathrm{X}$ Genomics ${ }^{2}$. These resulting multiple types of profiles: histology, spatial location, and gene expression, provide novel insights into the organization of cells and developmental biology, especially for the evolution of the tumor ${ }^{3-5}$. However, SRT data analysis for biology discovery remains challenging due to its low throughput, little sensitive, much sparse and noisy ${ }^{6,7}$.

Recently, several computational methods have been designed to analyze SRT data ${ }^{6}$. For example, Giotto used a similar processing strategy to single-cell RNA-seq (scRNA-seq), for feature selection, dimension reduction, and unsupervised clustering ${ }^{8}$. BayesSpace adopted a fully Bayesian statistical method to enhance the spatial measurement by using spatial neighborhood structure for clustering analysis ${ }^{7}$. SpaGCN adopting a graph convolutional network (GCN) approach integrated gene expression, spatial location, and histology to identify 
spatial domains and spatially variable genes (SVGs) ${ }^{9}$. stLearn integrated features of histology with spatial location to normalize gene expression data, and followed by clustering ${ }^{10}$. While these methods have many interesting findings, the lack of visual features that can be effectively and globally extracted from histology, efficient multi-view information fusion, and the biological contexts such as global positional information within a tissue, limits their disentangling capabilities in developmental biology.

On the other hand, GCN-based models have appeared as powerful tools to learn the representations of scRNA-seq data (i.e., by scGNN) ${ }^{11}$ and SRT data (i.e., by SpaGCN) ${ }^{9}$, however, these methods usually study networks with a single type of proximity between nodes, namely single-view network. Although SpaGCN proposed an RGB signal averaging strategy to convert histological data into the same measurement space as 2D space in the tissue slice as a third dimension before calculating the similarity between any two spots, to a certain extent we believe that this strategy discards the texture information in each spot. Besides, in SRT studies, $K$-nearest spots that are physically closest to the central spot are not necessarily the same as those that are the most histologically similar to the spot, and the distance evaluation metrics between multi-view data are also not the same, thus yielding networks with multiple views. Moreover, the contributions of neighboring spots to determine the cell type to which the central spot belongs are not identical, which is consistent with the assumption of graph attention network (GAT) ${ }^{12,13}$. More importantly, the quality of the information in different views may be different, hence, it would be preferable that one novel model can learn the representations for each view by GAT, and meanwhile collaboratively integrate multiple networks to learn robust representations by automatically training the weights of different views ${ }^{14}$.

We reason that (i) cells belonging to the same cell type but distributed in different areas and interacting with different cell types in the tissue may have different cell-states ${ }^{6}$; (ii) the determination of each cell-type (or sub-cell-type) to which each cell belongs, is related to its size, shape, and arrangement (i.e., tightness or looseness), hence the texture data of histology has rich information to characterize cell-state ${ }^{15}$; and (iii) the colors of the antibodies on the immunofluorescence staining of the tumor sample can roughly mark the tumor position in the tissue, yielding cell segmentation indicating biological contexts related to the tumor development.

In this work, we introduced stMVC (Spatial Transcriptomics data analysis by Multiple View Collaborative-learning), a framework that integrates four-layer information to elucidate tissue 
heterogeneity by attention-based multi-view graph collaborative learning, i.e., histology, gene expression data, spatial location, and cell segmentation (i.e., tumor position) indicating biological contexts. The unique features of stMVC are as follows: (i) for each spot, globally learning efficient visual features while removing artifacts from histology by data augmentations and contrastive learning; (ii) learning robust representations for each spot by training the weights of multi-view graphs including histological similarity graph (HSG) by visual features and spatial location graph (SLG) by physical coordinates, as well as the encoding of gene expression data as a feature matrix, via attention-based collaborative learning strategy, in a semi-supervision manner from cell segmentation; and (iii) for samples of human ovarian endometrial adenocarcinoma (OEAD) and breast invasive ductal carcinoma (IDC), identifying novel cancer-related cell-states (i.e., stemness, migration, and metastasis) missed by competing methods, and also transition cell-states, which were further validated by clinical data from other independent studies, demonstrating potential clinical and prognostic applications from SRT data. In particular, such a multi-view graph collaborative learning method is a flexible framework which is able to integrate not only SRT data from multi-sources or spatial multi-omics data but also spatial epigenomics or proteomics data.

\section{Results}

\section{Overview of stMVC model}

stMVC collaboratively integrated multiple graphs containing HSG by histological data $(I)$ and SLG by spatial locations $(S)$, through the semi-supervision learning from biological contexts $(Y)$ indicated by cell segmentation (see Manual cell segmentation) within a tissue, to clarify tissue heterogeneity (Fig.1a-d). Specifically, we (1) adopted the SimCLR model ${ }^{16}$ with the ResNet-50 as a base bone ${ }^{17}$ to extract visual features from histological images for each spot $v_{i}$ by maximizing the agreement between differently augmented views of the same spot image $\left(I_{i}\right)$, and then constructed HSG by the learned visual features (Fig.1b); (2) separately learned view-specific representations $\left(P_{i}^{1}\right.$ and $\left.P_{i}{ }^{2}\right)$ by semi-supervised graph attention autoencoder (SGATE) for each of two views: HSG and SLG, as well as the encoding of gene expression data by the autoencoder-based framework as a feature matrix (see Supplementary methods), and meanwhile collaboratively integrated two graphs to learn robust representations $\left(R_{i}\right)$ by attention mechanism, in a semi-supervision manner from cell segmentation (Fig.1c). Hence, $R$ is a low-dimensional feature representing the variation of these four-layer profiles, which is used to represent each spot as a point in the low-dimensional space, to do spatial clustering and 
visualization, where each cluster is considered as a spatial domain. In this work, for each spot, we aggregated gene expression data by its 15 nearest neighboring spots that are calculated based on the distance of robust representations $\left(R_{u}\right.$ and $\left.R_{v}\right)$ between any two spots $(u$ and $v)$, and then identified SVGs that are over-expressed in a domain by differential expression analysis (Fig.1d).

To emphasize the advantages of stMVC, we designed several comparative methods in our experiments: (i) the mean of all view-specific representations was used as a comparison to assess the efficiency of automatically learning the weights of different views by attention, which is named stMVC-M; (ii) SGATE was used to learn view-specific representations for either SLG (SGATE-SLG) or HSG (SGATE-HSG) to assess the effectiveness of multi-view graph collaborative learning; and (iii) SGATE for naïve HSG (SGATE-HSG-N) created by the visual features extracted from the ResNet-50 model pre-trained by ImageNet ${ }^{18}$ was used to evaluate the quality of the visual features learned by our trained SimCLR model.

stMVC is robust to the proportion of labels for the training, which was evaluated based on 12 slices of the human dorsolateral prefrontal cortex (DLPFC) dataset with annotations from the previous study ${ }^{19}$. Specifically, for each slice, we (i) randomly selected the spots with labels, with the proportion ranging from 0.1 to 0.9 by 0.1 , thus generating nine label datasets; (ii) randomly selected $70 \%$ of spots as the training set, the labels of which from one of nine datasets were used to supervise the training of stMVC, stMVC-M, and all SGATE-based single-view models; and (iii) for each model, predicted the cell clusters by the Louvain algorithm, and assessed the influence of the proportion of labels on the model training via clustering accuracy including Adjusted Rand Index (ARI) and Normalized Mutual Information (NMI) by comparing predicted cell types with the annotation. Overall, we observed that the clustering accuracy of all models slightly increases with the proportion of labels for the training, and almost all models have a higher accuracy at the training with $70 \%$ labels. Hence, we treated $70 \%$ as a cutoff to select labels for model training. Additionally, we found that stMVC achieves higher and comparable performance than stMVC-M and SGATE-SLG, these three models perform better than that by two HSG-based models, and SGATE-HSG performs better than SGATE-HSG-N (Supplementary Fig.1). Overall, these results indicate the efficiency of stMVC.

\section{stMVC revealed the evolutionary relationship between different spatial domains}


The most important feature of the stMVC model is that the learned low-dimensional representations can reveal the relationship between different spatial domains in a semisupervision manner from biological contexts. To assess the performance of stMVC, we applied it to process 12 DLPFC slices, each of which was manually annotated with six layers and white matter (WM), and the known evolutional relationship between these layers is WM -> Layer6-> Layer5 -> Layer4 -> Layer3 -> Layer2 -> Layer1 (Fig.2a) ${ }^{19}$. By default, through the semisupervised learning of $70 \%$ of the annotation, stMVC, stMVC-M, and all SGATE-based single-view models extracted 10-dimensional features from the input. For comparison, we also applied four recently developed methods including SpaGCN, BayesSpace, Giotto, and stLearn for spatial clustering or visualization with the default parameters. Subsequently, we predicted cell clusters by the Louvain algorithm, evaluated clustering accuracy based on ARI and NMI by comparing the predicted cell types with the annotation, and then visualized these lowdimensional features by mapping them into two uniform manifold approximation and projection (UMAP) spaces.

In summary, slice 151673 as a representative example (Fig.2a,b), we found that (i) the clustering accuracy of stMVC is higher than that by all SGATE-based single-view models, SpaGCN, BayesSpace, Giotto, and stLearn, indicating that our multi-view graph collaborative learning model did capture more useful information than that from single-view graph model; (ii) stMVC performs better than stMVC-M in terms of detecting Layer4 and Layer6, demonstrating that attention-based strategy is able to focus on finer structure than that by meanbased strategy; (iii) SGATE-HSG performs better than SGATE-HSG-N, which indicates that our trained SimCLR model with ResNet-50 by data augmentations and contrastive learning did learn more rich visual features than those from naïve ResNet-50 model pre-trained by ImageNet; and (iv) each spatial domain is assigned almost by different feature embeddings from stMVC, stMVC-M and SGATE-SLG model, compared to Giotto, stLearn, and two HSGbased models, particularly the distance between different domains of our stMVC model can reflect the trajectory of development, which shows that our stMVC model can clarify the complex relationship between different spatial domains. In addition, we further applied the stMVC model with the same network structure and hyperparameters as the above model for the other 11 samples to check its efficiency, and observed that both ARI and NMI scores of the stMVC model are significantly higher than those by all SGATE-based single-view models, stLearn, and BayesSpace (Fig.2c). 
Similar to our previous studies ${ }^{20,21}$, we further assessed the quality of the denoised data from stMVC by our defined AGI scores based on clustering and the expression levels of both marker and housekeeping genes (see Supplementary methods). We observed that stMVC and stMVC-M have comparable performance and perform slightly better than all SGATE-based single-view models and BayesSpace, and stLearn performs worst (Fig.2d). For instance, some known layer marker genes such as SNAP25, MOBP, KRT17, PCP4, PVALB, ENC1, and CCK are more specifically expressed in their corresponding spatial location, compared with those by other models. (Fig.2e and Supplementary Fig.2).

\section{stMVC contributed to detecting novel cell-states missed by competing methods}

To illustrate stMVC is able to clarify cancer cells that are distributed at different positions in the tissue, we further analyzed the OEAD sample (i.e., ovarian cancer). We annotated 18 regions based on our segmentation strategy detailed in Manual cell segmentation (Fig.3a and Supplementary Fig.3a), treated the regions as rough labels, and randomly selected $70 \%$ of which to supervise the training of the stMVC model. stMVC, stMVC-M, and all SGATE-based single-view models extracted 18-dimensional features from the input. For comparison, we also applied Giotto, stLearn, and BayesSpace for spatial clustering or visualization. Subsequently, we predicted cell clusters and visualized these low-dimensional features by mapping them into two UMAP spaces.

We found that stMVC facilitates detecting more domains enriched for the cancer regions, compared to those by all SGATE-based single-view models, stMVC-M, Giotto, stLearn, and BayesSpace (Fig.3b,c and Supplementary Fig.3b-d). Particularly for the region outlined with black color, we noted that stMVC detects four domains while Giotto, stLearn, and BayesSpace detect one domain, and the feature embeddings extracted by stMVC are better separated between four different domains than those by Giotto and stLearn (Fig.3b-d).

To further support the accuracy of detecting novel cell-states missed by other methods, taking the above described four domains as an example, we adopted three independent ways. Specifically, we found that (i) four clusters have different functions: cluster 10 cells show various biological functions such as gluconeogenesis ${ }^{22}$ and NF-kB signaling pathway promoting cancer stem cell maintenance, metastasis, and immune evasion ${ }^{23}$; cluster 12 cells participate in Wnt signaling pathway, cell growth, cell proliferation, and cell migration; cluster 13 cells are related to Toll-like receptor signaling pathway and cell differentiation; cluster 11 cells are in involved in a HIF-1 signaling pathway playing an import role in tumor adaptation 
to microenvironmental hypoxia (Fig.3e); (ii) GRPC5A (for cluster 12) and SPP1 (for cluster 11) are enriched in different cell populations that are not clearly separated in the scRNA-seq data of 4,081 epithelial cells from an ovarian cancer patient ${ }^{24}$ (Fig.3f), indicating that these cells exist in ovarian cancer, as well as the advantage of SRT data over scRNA-seq data in terms of visualization of the gene expression levels in the context of tissue; and (iii) the expression of GRPC5A ( $\mathrm{p}=0.028)$ and SPP1 $(\mathrm{p}=0.0081)$ is significantly correlated with shorter overall survival, which was evaluated by ovarian cancer from The Cancer Genome Atlas (TCGA) database (Fig.3g). In addition, we noted that cluster 10 cells display elevated expression of S100A9 ${ }^{25}$ and PNOC ${ }^{26}$, both of which are reported to be significantly higher in high-grade serous ovarian tumors relative to the normal ovary, and cluster 13 cells exhibit overexpression of NEDD9 which is associated with the progression of and an unfavorable prognosis in ovarian cancer ${ }^{27}$. In short, stMVC is more conductive to clarify tissue heterogeneity in terms of tumor cells related to stemness, cell proliferation, and migration in different spatial regions, as well as their clinical and prognostic applications.

\section{stMVC enabled us to elucidate intratumoral heterogeneity in IDC}

To further demonstrate the application power of stMVC, we applied it to analyze the IDC sample of breast cancer by stMVC to elucidate its intratumoral heterogeneity and infer possible trajectories in cancer development. IDC sample was annotated with three types of tumor regions: invasive carcinoma (IC), carcinoma in situ (CIS), benign hyperplasia (BH) (Fig.4a) by the previous study ${ }^{7}$. We manually defined 16 regions based on our segmentation strategy (Supplementary Fig.4a), and randomly selected $70 \%$ of spots per region as the spots having labels to train stMVC. By default, stMVC, stMVC-M, and all SGATE-based single-view models extracted 16-dimensional features from the input. For comparison, we also applied Giotto, stLearn, and BayesSpace to process the IDC sample. Afterward, we predicted cell clusters and visualized these latent features by mapping them into two UMAP spaces.

In summary, we found that (i) stMVC and stMVC-M tend to detect more domains enriched for tumor regions, especially for the CIS region, and the feature embeddings of the domains from both methods are better separated, compared with those from all SGATE-based single-view models, BayesSpace, stLearn, and Giotto (Fig.4b,c and Supplementary Figs.4b,c and 5), indicating that tumor cells in different spatial regions have different cell-states, which is consistent with the observation from OEAD sample; (ii) elevated expression of HER2 and ER throughout the tumor regions and minimal expression of PR for the data from stMVC, which 
is in line with the clinical report of Luminal B (Fig.4d and Supplementary Fig.6); (iii) overexpression of CD45 throughout non-tumor region indicates the region is related to immune, specifically, cluster 1 is enriched for the signatures of T (CD4, CD8A), Macrophage (CD14), and DC (HLA-DRB1) cells; cluster 2 is enriched for Plasma B (IGCL2 and IGHG3) and two types of endothelial cells (FABP4 for endothelial_DCN, VWF and ENG for endothelial_VWF) defined by our previous study ${ }^{28}$; cluster 7 is enriched for Myofibroblast (MYLK) and Follicular B cells (MS4A1). Compared with the result by BayesSapce ${ }^{7}$, stMVC enables detecting the finer structure of the tumor microenvironment (Fig.4e and Supplementary Fig.6); and (iv) elevated expression of EPCAM is enriched in the tumor region with higher HER2 while over-expression of MUC1 is enriched in the ER-positive tumor region (Fig.4f), which is in accordant with the observation from scRNA-seq data of 20 breast cancer patients (Fig.5a) 29. Besides, stMVC and stMVC-M have similar expression patterns, and perform better than those by stLearn and BayesSpace (Supplementary Fig.6).

More interestingly, we observed that stMVC detects four clusters in the ER-positive IC region outlined with red color, the detection of clusters 10 and 3 are very different from Giotto, stLearn, and BayesSpace (Figs.4a,b and 5b, Supplementary Fig.7); and there is a possible trajectory between three clusters, i.e., cluster 6 -> cluster $10->$ cluster 3 (Fig.4c). We validated our predictions by the following several independent datasets. Specifically, we observed that (i) four clusters have different functions, i.e., cluster 6 cells exhibit a variety of biological functions such as activation of Stat 3 protein to modulate cancer stemness ${ }^{30}$; cluster 10 cells are related to cell adhesion and cell division; cluster 3 cells participate in cell growth and proliferation; and cluster 5 cells mediate cell migration and apoptotic process (Supplementary Fig.8); (ii) by re-analyzing 24,489 epithelial cells from 20 breast cancer patients, the higher expression of ARMT1, SCUBE2, and CCNO is in scRNA-seq data from ER-positive patients, compared with those from ER-negative patients (Figs.4f and 5a), which is consistent with the previous conclusion: ER and ARMT1 are co-expressed, and ARMT1 affects cell proliferation 31, as well as clusters 10 and 3 cells existing in breast cancer; (iii) there is a trajectory between three clusters identified from 2,352 epithelial cells of CID4067 (a representative luminal B patient) through analyzing its scRNA-seq by Monocle $2^{32}$, and with the estimated pseudo-time, the expression of PDZK1IP1 for cluster 6 decreases, ARMT1 and SCUBE2 for clusters 10 and 3 increase, supporting our trajectory inference between clusters 6, 10, and 3 (Fig.5c); and (iv) the expression of CCNO $(p=0.044)$ and SCUBE2 $(p=0.0018)$ is significantly correlated with shorter overall survival, which was evaluated by ER-positive breast cancer patients from 
TCGA database (Fig.5d). Furthermore, over-expressed PDZK1IP1 and LUCAT1 in cluster 6 are related to regulating breast cancer cell stemness ${ }^{33,34}$, hence, these results indicate that there is possible cell development from cancer stem cells to malignant cells. In addition, cluster 5 cells displayed elevated expression of MMP11 (Supplementary Fig.7), a biomarker for metastatic breast cancer ${ }^{35,36}$. Moreover, the SVGs by stMVC-M were almost the same as those by stMVC (Supplementary Figs.5 and 7). Taken together, stMVC can identify cancer-related cell-states and transition cell-states while supporting clinical and prognostic cancer applications from SRT data.

\section{Discussion}

In this work, we proposed stMVC for analyzing SRT data to disentangle the heterogeneity of tissue, especially for the tumor, which integrates four-layer profiles: histological image data, spatial location, gene expression, and cell segmentation (i.e., global position) indicating biological contexts, by attention-based multi-view graph collaborative learning. To our best knowledge, this is the first model to use such global position information of tissue in analyzing SRT data. Unlike previous methods that integrate histology and spatial location data by a userdefined weight, for example, SpaGCN manually adjusts the weight of histology in gene expression smoothing, stMVC adopts an attention-based strategy to automatically learn weights of different views for robust representations. Besides, our SimCLR model trained for histological image data by data augmentations and contrastive learning did help stMVC to learn more efficient visual features, compared with those from the pre-trained ResNet-50 mode by ImageNet, which was used by stLearn. The evaluations on two real cancer datasets demonstrated the unique advantages of stMVC described above, which are able to detect novel cell-states related to cell stemness, migration, and metastasis distributed in different spatial domains, providing novel biological insights into tumor heterogeneity. In particular, for the breast cancer dataset, we demonstrated potential clinical and prognostic applications from SRT data, by identifying novel cancer-related cell-states and also transition cell-states missed by competing methods, which were further validated by clinical data.

By comparing stMVC with all SGATE-based single-view models, we found that stMVC has a better performance in terms of clustering, inference of trajectory, and denoising, which is mainly attributed to the collaborative learning of multi-view graphs. Besides, we observed that SGATE-SLG performs better than SGATE-HSG, however, SGATE-HSG is able to capture some rich boundary information as a complementary to SGATE-SLG. Hence, we believed that 
the perspective of modeling SRT data by multi-view graphs enables a better understanding of the tissue heterogeneity, compared with that by single-view graph.

In addition, by comparing with stMVC-M, we noted that stMVC achieves better and comparable performance. Specifically, (i) regarding histology without rich texture information, such as DLPFC and OEAD samples, stMVC-M is more vulnerable to the noise signals from the histological visual features while stMVC is easier to capture the finer structure by automatically learning the weight of each of multiple graphs; (ii) regarding histology with rich tissue anatomical structure, like IDC sample, both models have a similar result. Hence, we also implemented mean-based strategy in stMVC model as an option for users to select.

As of now, developing models for integrating SRT data from multiple samples are facing several challenges, for example, the batch effect of gene expression data from multiple sources, as well as their sparsity and noise; constructing the association between spots from different physical metric spaces; and removing the artifacts of histology while creating the relationship between multiple samples ${ }^{37,38}$. However, we believed that (i) the visual feature extraction model by data augmentations and contrastive learning provides a solution to construct the association of spots between different samples; and (ii) the multi-view graph collaborative learning model can provide a novel perspective to integrate multiple SRT datasets by combining multi-layer profiles data. Besides, stMVC is easily scalable to process spatially resolved chromatin accessibility (ATAC-seq) or proteomics data ${ }^{39,40}$, by replacing the feature matrix from gene expression data with that from ATAC-seq or proteomics data. Furthermore, with the advance of spatial multi-omics technology ${ }^{41}$, stMVC can be easily adjusted to adapt by either adding more graphs created by different omics data or substituting the feature matrix from single-omics data with that from multi-omics data fusion.

Some limitations still are found in stMVC: (i) compared with the visual features extraction framework from the ResNet-50 model pre-trained by the ImageNet, the 500 iterations training of SimCLR needs more computational resources and times; and (ii) cell segmentation for tumor position is manually annotated based on the staining density of antibodies. With the exploration of deep learning frameworks of generalizable cell segmentation tools ${ }^{42,43}$, we will further investigate creating a more efficient stMVC model with a more automatic architecture in the future study.

\section{Methods}

\section{stMVC model}


stMVC is a multi-view graph collaborative learning model, which integrates four-layer profiles: histological image data $(I)$, spatial location $(S)$, gene expression $(X)$, and manual cell segmentation $(Y)$ to elucidate tumor heterogeneity (Fig.1a-d). Specifically, we (1) learned visual features $\left(h_{i}\right)$ for each spot $(i)$ by SimCLR with feature extraction framework from ResNet-50, and then constructed HSG based on $h_{i}$; and (2) captured the spot proximities encoded in either histology or spatial location, as well as the encoding of gene expression data as a feature matrix, and then simultaneously integrated these two graphs for the robust representations by automatically learning the weight of different graphs via attention, in a semisupervision manner from cell segmentation.

\section{Learning visual features by contrastive learning to construct HSG}

To remove the noise from histology staining and extract morphological information from histological image data, we adopted SimCLR model to efficiently learn visual features for each spot by maximizing agreement between differently augmented views of the same spot image via a contrastive loss in the latent space (Fig.1b). For a spot $v_{i}$, the physical location of which in the tissue slice is represented by $\left(s_{x i}, s_{y i}\right)$, a square containing $40 \times 40$ pixels centered on $\left(s_{x i}, s_{y i}\right)$ is regarded as its spot image $I_{i}$. The specific model is described as follows:

(1) for each spot image $I_{i}$, we applied a stochastic data augmentation module to randomly transform it into two correlated views for the same spot image $I_{i}$, denoted as $I_{m}{ }^{\prime}$ and $I_{n}{ }^{\prime}$, and considered these two views as a positive pair. In this work, we sequentially apply two simple augmentations: random cropping followed by resizing back to the original size, and random color distortions including randomly changing the brightness, contrast, saturation, and hue of an image ${ }^{16}$.

(2) we adopted the ResNet-50 framework as a base encoder $f(\cdot)$ to extract features $\left(h_{m}\right.$ and $h_{n}$ ) from two augmented views $\left(I_{m}{ }^{\prime}\right.$ and $\left.I_{n}{ }^{\prime}\right)$, where $h_{m}, h_{n} \in R^{2048}$ is the output after average pooling layer.

(3) we used a Multilayer Perceptron (MLP) with one hidden layer to obtain latent features $l_{m}=$ $g\left(h_{m}\right)=W^{(2)} \sigma\left(W^{(1)} h_{m}\right)$ where $\sigma$ is a 'relu' function, the first and second layers are set as 512 and 128 , respectively, and $l_{m}$ is used to define contrastive loss.

(4) Given a set $\left\{I_{k}\right\}$ including a positive pair of examples $I_{m}$ and $I_{n}$, the contrastive prediction task aims to identify $I_{n}$ in $\left\{I_{k}\right\}_{k \neq m}$ for a given $I_{m}$. The loss function for a positive pair of samples $\left(I_{m}{ }^{\prime}, I_{n}{ }^{\prime}\right)$ is defined as: 


$$
l_{i, j}=-\log \frac{\exp \left(\operatorname{sim}\left(l_{m}, l_{n}\right) / \tau\right)}{\sum_{k=1}^{2 N} \mathbb{I}_{[k \neq m]} \exp \left(\operatorname{sim}\left(l_{m}, l_{n}\right) / \tau\right)}
$$

where $\operatorname{sim}\left(l_{m}, l_{n}\right)=\frac{l_{m}{ }^{\mathrm{T}} l_{n}}{\left\|l_{m}\right\|\left\|l_{n}\right\|}, \mathbb{I}_{[k \neq m]} \in\{0,1\}$ is an indicator function evaluating to 1 if $k \neq m$, $\tau$ denotes a temperature parameter, and $N$ indicates batch size.

For each SRT data, we randomly selected $85 \%$ of the spots as the training sets to train the SimCLR model, and the remaining $15 \%$ of the spots as the testing set to test it. Adam optimizer with both a $1 e^{-6}$ weight decay and a $1 e^{-4}$ learning rate is used to minimize the above loss function. After 500 iterations of model training, we have SimCLR finished, and then calculated the histological similarity between any two spots by calculating the Cosine similarity between learned features $h_{u}$ and $h_{v}$ from spots $u$ and $v$ as follows: $\cos \left(h_{u}, h_{v}\right)=\frac{h_{u} \cdot h_{v}}{\left\|h_{u}\right\|\left\|h_{v}\right\|}$. A Sixnearest neighbor graph for each spot is kept constructing the HSG $G_{1}=\left(V, E_{1}\right)$, where each vertex $v \in V$ indicates a spot, and every two vertices in $V$ are connected by an edge $e \in E_{1}$. As a comparison, we used the same strategy to construct naïve $\operatorname{HSG} G_{1}{ }^{\prime}=\left(V, E_{1}{ }^{\prime}\right)$ by the feature extracted by feature extraction framework of ResNet-50 pre-trained by ImageNet to validate the effectiveness of our trained SimCLR model.

\section{Learning robust representations by multiple view graph collaborative learning}

We used SGATEs for learning view-specific representations in stMVC, where a SGATE for each view was trained under weak supervision of the cell segmentation to capture its efficient low-dimensional manifold structure by integrating gene expression with either $\operatorname{HSG}\left(G_{1}\right)$ or SLG, $\left(G_{2}=\left(V, E_{2}\right)\right)$ which is constructed by a six-nearest neighbor graph for each spot based on the Euclidean distance of their physical location in the tissue slice ${ }^{44}$, and meanwhile collaboratively integrated them to learn robust representations for each spot (Fig.1c). In this work, two graphs $\left(G_{1}\right.$ and $\left.G_{2}\right)$ have the same vertex (spot) set but different edges.

stMVC for learning view-specific representations. For each view graph, a SGATE model aims to learn accurate low-dimensional features with the following inputs: an adjacency matrix $\left(A_{m} \in R^{n \times n}\right)$ representing $m$ th graph $G_{m}$, cell labels indicated by cell segmentation $(Y)$, and feature matrix $Z \in R^{n \times d}$ learned from gene expression $(X)$ by autoencoder-based framework (see Supplementary methods), where $n$ is the number of spots, $d$ is the number of latent features and the default value of which is 50 , and $m \in\{1,2\}$. A GAT can be built by stacking multiple multi-head graph attention layers ${ }^{13}$. Specifically, each layer is defined as: 


$$
\begin{gathered}
h_{i}{ }^{(l+1)}=\sigma\left(\frac{1}{K} \sum_{k=1}^{K} \sum_{j \in N_{i}} \alpha_{i j}{ }^{k} W^{k} h_{j}{ }^{l}\right) \\
\alpha_{i j}{ }^{k}=\frac{\exp \left(\operatorname{LeakyReLU}\left(\left(a^{k}\right)^{T}\left[W^{k} h_{i}{ }^{l} \| W^{k} h_{j}{ }^{l}\right]\right)\right)}{\sum_{m \in N_{i}} \exp \left(\operatorname{LeakyReLU}\left(\left(a^{k}\right)^{T}\left[W^{k} h_{i}{ }^{l} \| W^{k} h_{m}{ }^{l}\right]\right)\right)}
\end{gathered}
$$

where $K$ indicates the number of head attention and the default value is two, $\alpha_{i j}{ }^{k}$ is normalized attention coefficients computed by the $k$ th attention mechanism $\left(a^{k}\right), W^{k}$ is the corresponding input linear transformation's weight matrix, $N_{i}$ is the neighborhood of spot $\left(v_{i}\right)$ in the graph, and $h_{j}{ }^{l}$ is the input feature of node $j$ of the $l$ th layer.

The encoder of each GATE model is composed of two layers of GAT, the first and second layers are set at 25 and 10 (36 and 18, or 32 and 16), respectively. The graph embedding for each graph is represented by $P_{m}$. The decoder of each GATE model is defined as an inner product between the embedding:

$$
A_{m}{ }^{\prime}=\operatorname{sigmoid}\left(P_{m} P_{m}{ }^{T}\right)
$$

where $A_{m}{ }^{\prime}$ is the reconstructed adjacency matrix of $A_{m}$. The goal of learning each GATE model is to minimize the cross-entropy between the input adjacency matrix $A_{m}$ and $A_{m}{ }^{\prime}$ :

$$
L_{\text {recon-m }}=-\frac{1}{n \times n} \sum_{i=1}^{n} \sum_{j=1}^{n}\left(a_{m i j} \times \log \left(a_{m i j}{ }^{\prime}\right)+\left(1-a_{m i j}\right) \times \log \left(1-a_{m i j}{ }^{\prime}\right)\right)
$$

where $a_{m i j}$ and $a_{m i j}{ }^{\prime}$ are the elements of the adjacency matrix $A_{m}$ and $A_{m}{ }^{\prime}$ in the $i$ th row and the $j$ th column of $m$ th graph, respectively.

To capture biological contexts in the tissue, we further extended the use of the GATE model through one layer with $C$ nodes to do the prediction $Y_{m}{ }^{\prime}=\operatorname{softmax}\left(W_{m}{ }^{(1)} P_{m}\right)$ in a semisupervision manner from cell segmentation, and the loss function of which is summarized as follows:

$$
L_{\text {pre }-m}=\frac{1}{S} \sum_{l=1}^{S}\left(-\sum_{i=1}^{C} y_{i} \log \left(y_{m i}{ }^{\prime}\right)\right)
$$

where $S$ is the number of labeled samples, $C$ is the number of classes, and $y_{i}$ and $y_{m i}{ }^{\prime}$ are the label vector of spot $v_{i}$ from the cell segmentation and the prediction of the $m$ th graph, respectively.

Taken together, the loss function of each SGATE model is summarized as: 


$$
L_{S G A T E-m}=L_{\text {recon-m }}+\beta L_{\text {pre-m }}
$$

Where $\beta$ is a parameter used to control the weight of two loss functions, and the default value is eight.

stMVC for multi-view graph collaborative learning. After learning view-specific representations $P_{i}{ }^{m}$ for spot $\left(v_{i}\right)$ by the $m$ th SGATE model, we applied the collaborative learning model to integrate different view graphs for its robust representations $\left(R_{i}\right)$. The contribution of each view to $R_{i}$ is unavailable, hence, we proposed the attention-based strategy to learn the weight of each graph for the final representations by the following function:

$$
R_{i}=\sum_{m=1}^{M} \gamma_{i}^{m} P_{i}^{m}
$$

where $M$ is the number of views. Inspired by attention based models ${ }^{14,45}$, we defined the weight of one view for node $v_{i}$ using the following function:

$$
\gamma_{i}^{m}=\frac{\exp \left(a_{1} \cdot P_{i}{ }^{C}\right)}{\sum_{m=1}^{M} \exp \left(a_{m} \cdot P_{i}{ }^{C}\right)}
$$

Where $P_{i}^{C}$ is the concatenation of all view-specific representations of spot $v_{i}$, and $a_{m}$ is feature vector of the $m$ th view, describing what kinds of spots will consider the $m$ th view as informative. If $P_{i}{ }^{C}$ and $a_{m}$ have a large dot product, meaning spot $v_{i}$ believes that the $m$ th view is an informative view, and vice versa.

To collaboratively integrate different views into the same semantic space ${ }^{21}$, we further leveraged robust representations to fine-tune the learning of each view graph by transferring knowledge from robust representations to each view-specific representations, as well as optimizing the parameters of $\left\{a_{m}\right\}_{m=1}^{M}$ in semi-supervised manner through a MLP with one hidden layer to do spot class prediction $Y_{C L}{ }^{\prime}=\operatorname{softmax}\left(W^{(2)} \sigma\left(W^{(1)} R\right)\right)$. The corresponding loss functions are defined as follows:

$$
\begin{aligned}
& L_{\text {transfer }}=\sum_{i=1}^{n} \sum_{m=1}^{M} \gamma_{i}^{m}\left(R_{i}-P_{i}{ }^{m}\right)^{2} \\
& L_{\text {pre }-C L}=\frac{1}{S} \sum_{l=1}^{S}\left(-\sum_{i=1}^{C} y_{i} \log \left(y_{C L i}{ }^{\prime}\right)\right)
\end{aligned}
$$

In summary, the total loss function for robust representations by collaboratively integrate multiple views is summarized as follows: 


$$
L_{M V C}=\sum_{m=1}^{M} L_{\text {recon-m }}+\beta^{\prime} \sum_{m=1}^{M} L_{\text {pre-m }}+\delta L_{\text {transfer }}+\varphi L_{\text {pre }-C L}
$$

Where $\beta^{\prime}, \delta$ and $\varphi$ are used to control the weights of these regularization terms, and the default values of them are 10,0.0005, and 100, respectively.

Overall, the objective function of our stMVC model can be effectively optimized with the following iterations, specifically, for each iteration, we (i) optimized the parameters of each SGATE model to learn the view-specific representations based on equation (7), as well as equation (10) when the robust representations $(R)$ has been inferred by the equation (8), and followed by inferring $R$ via equation (8); and (ii) optimized the parameters of all SGATE models and the parameter vectors of all graphs based on equation (12), and then inferred $R$ based on the optimized stMVC model. After the convergence, we have the stMVC model trained, and $R$ was used for the spatial clustering, visualization, and denoising (Fig.1d).

\section{Datasets and preprocessing}

SRT data. In our study, three SRT datasets with paired gene expression and histology were generated from the 10X platform. Specifically, (i) human DLPFC dataset with 12 slices that each contains the number of spots ranging from 3,460 to 4,789 with a median of 3,844 , and is manually annotated to the six cortical layers and WM by the previous study ${ }^{19}$. Besides, we randomly selected manual annotation of each of 12 slices, with the proportion ranging from 0.1 to 0.9 by 0.1 , thus generating nine manual annotation sets for each slice, which were used to check if or not stMVC can capture the inner structure of tissue by few labels; (ii) human OEAD sample with matching gene expression (3,493 spots) and immunofluorescence staining with an anti-human CD45 antibody and DAPI; and (iii) human IDC sample with paired gene expression data $(4,727$ spots) and immunofluorescence staining with an anti-human CD3 antibody and DAPI. Both OEAD and IDC samples were downloaded from the 10X website 46,47 .

scRNA-seq and bulk RNA-seq data. To validate our predictions for the sample of ovarian cancer, we collected 4,081 epithelial cells from one ovarian cancer patient from a previous study ${ }^{24}$. Besides, 24,489 epithelial cells were collected from 20 breast cancer patients from previous research, including five luminal $\mathrm{B}$, three Luminal A, three HER2-positive, seven triple-negative breast cancers (TNBCs), one HER2-positive and ER-positive, and one normal ${ }^{29}$, to support our novel findings, and the subtypes are determined by their clinical reports and predictions. Furthermore, the bulk RNA-seq data and clinical information of both ovarian and 
breast cancer of TCGA database were downloaded from the Xena platform for survival analysis ${ }^{48}$, and the PAM50 subtype classifications of breast cancer of TCGA were collected from previous research ${ }^{49}$.

Preprocessing. To comprehensively compare each computational method on these three datasets, for each gene expression data, the top 2,000 highly variable genes (HVGs) by the 'vst' method of Seurat ${ }^{50}$, were used for comparison. Besides, to efficiently capture the information within gene expression data, we mapped each data into 50-dimensional latent features based on our designed autoencoder-based framework (see Supplementary methods), and took them as the input of stMVC.

\section{Manual cell segmentation}

We designed a strategy to define biological contexts in each tissue based on the following assumptions: (1) the colors of the antibodies on the immunofluorescence staining of the tumor histology can roughly define the tumor location; and (2) cells belonging to the same cell type but are separated by other cells such as immune or other mesenchymal cells may have different cell states. After annotating different regions in the histology image by labelme software ${ }^{51}$, we further applied the OpenCV package ${ }^{52}$ to determine each region (or context) to which each spot $\left(v_{i}\right)$ belongs, by calculating the proportion between area of the intersection between a square $\left(I R_{i}\right)$ containing $40 \times 40$ pixels centered on $\left(s_{x i}, s_{y i}\right)$ and a region $\left(R_{j}\right)$, compared to the square. The function is defined as follows:

$$
\text { Proportion }=\frac{\text { Intersection }\left(I R_{i}, R_{j}\right)}{I R_{i}}
$$

By setting Proportion $\geq 0.5$, for the OEAD sample, 1,658 spots were separately classified into 17 different regions in the tumor, and the remaining 1,835 spots in the non-tumor region were treated as the 18th region (Supplementary Fig.3), and for the IDC sample, 2,091 spots were classified into 15 different regions in the tumor, and the remaining 2,636 spots were regarded as the 16th region (Supplementary Fig.4).

\section{Clustering and Visualization}

After applying stMVC in analyzing SRT data, we learned the accurate low-dimensional representations $(R)$ representing the relationship between any two spots. To further clarify tissue heterogeneity, given $R$ as the input, we adopted 'FindNeighbors' and 'FindClusters' function with default parameters from the Seurat package ${ }^{50}$ to determine k-nearest neighbors 
(KNNs) for each spot, construct the shared nearest neighbor (SNN) graph, predict the cell clusters by the Louvain algorithm, and each cluster is considered as a spatial domain.

We utilized the UMAP algorithm to map the low-dimensional features from each computational method to two-dimension, visualized the distance of cell embedding between different cell populations by 'Dimplot' function, and visualized the cell clustering and gene expression patterns at the spatial level by 'SpatialDimPlot', and 'SpatialFeaturePlot' function, respectively.

\section{Identification of SVGs}

We constructed KNN graph for each spot based on the learned low-dimensional representations $(R)$, and adopted the $\mathrm{KNN}$-smoothing algorithm to aggregate information from 15 nearest spots for each spot to denoise the gene expression data. Then, we identified SVGs from 2,000 HVGs among different spatial domains from the stMVC model by 'FindAllMarkers' from Seurat package.

\section{Evaluation of the clustering}

For the real datasets with known cell types, we utilized the ARI ${ }^{53}$ and NMI ${ }^{54}$ score to evaluate the clustering performance of each method.

\section{Data availability}

The datasets used in the study are publicly available from the Gene Expression Omnibus (GEO) repository under the accession number: GSE176078, EMBL-EBI under the accession number: E-MTAB-8859, Xena platform, and 10X Genomics Website.

\section{References}

1 Ståhl, P. L. et al. Visualization and analysis of gene expression in tissue sections by spatial transcriptomics. Science 353, 78-82 (2016).

2 GENOMICS, $\mathrm{x}$ Visium Gene Expession, $<$ https://www.10xgenomics.com/products/spatial-gene-expression $>$ (2021).

3 from Xiaowei, A. C. Method of the Year 2020: spatially resolved transcriptomics.

4 Hunter, M. V., Moncada, R., Weiss, J. M., Yanai, I. \& White, R. M. Spatial transcriptomics reveals the architecture of the tumor/microenvironment interface. bioRxiv (2020).

5 Liao, J., Lu, X., Shao, X., Zhu, L. \& Fan, X. Uncovering an organ's molecular architecture at single-cell resolution by spatially resolved transcriptomics. Trends Biotechnol 39, 43-58 (2021).

6 Dries, R. et al. Advances in spatial transcriptomic data analysis. Genome Res 31, 17061718 (2021). 
7 Zhao, E. et al. Spatial transcriptomics at subspot resolution with BayesSpace. Nature Biotechnology, 1-10 (2021).

8 Dries, R. et al. Giotto: a toolbox for integrative analysis and visualization of spatial expression data. Genome Biol 22, 1-31 (2021).

$9 \mathrm{Hu}$, J. et al. SpaGCN: Integrating gene expression, spatial location and histology to identify spatial domains and spatially variable genes by graph convolutional network. Nat Methods, 1-10 (2021).

10 Pham, D. et al. stLearn: integrating spatial location, tissue morphology and gene expression to find cell types, cell-cell interactions and spatial trajectories within undissociated tissues. bioRxiv (2020).

11 Wang, J. et al. scGNN is a novel graph neural network framework for single-cell RNASeq analyses. Nat Commun 12, 1-11 (2021).

$12 \mathrm{Wu}, \mathrm{Z}$. et al. A comprehensive survey on graph neural networks. IEEE transactions on neural networks and learning systems 32, 4-24 (2020).

13 Veličković, P. et al. Graph attention networks. arXiv preprint arXiv:1710.10903 (2017).

$14 \mathrm{Qu}, \mathrm{M}$. et al. in Proceedings of the 2017 ACM on Conference on Information and Knowledge Management. 1767-1776.

15 Gurcan, M. N. et al. Histopathological image analysis: A review. IEEE reviews in biomedical engineering 2, 147-171 (2009).

16 Chen, T., Kornblith, S., Norouzi, M. \& Hinton, G. in International conference on machine learning. 1597-1607 (PMLR).

17 He, K., Zhang, X., Ren, S. \& Sun, J. in Proceedings of the IEEE conference on computer vision and pattern recognition. 770-778.

18 Deng, J. et al. in 2009 IEEE conference on computer vision and pattern recognition. 248-255 (Ieee).

19 Maynard, K. R. et al. Transcriptome-scale spatial gene expression in the human dorsolateral prefrontal cortex. Nature neuroscience 24, 425-436 (2021).

20 Zuo, C. \& Chen, L. Deep-joint-learning analysis model of single cell transcriptome and open chromatin accessibility data. Brief Bioinform 22, bbaa287 (2021).

21 Zuo, C., Dai, H. \& Chen, L. Deep cross-omics cycle attention model for joint analysis of single-cell multi-omics data. Bioinformatics (2021).

22 Grasmann, G., Smolle, E., Olschewski, H. \& Leithner, K. Gluconeogenesis in cancer cells-Repurposing of a starvation-induced metabolic pathway? Biochimica et Biophysica Acta (BBA)-Reviews on Cancer 1872, 24-36 (2019).

23 Harrington, B. S. \& Annunziata, C. M. NF- $\kappa$ B signaling in ovarian cancer. Cancers 11, 1182 (2019).

24 Nelson, L. et al. A living biobank of ovarian cancer ex vivo models reveals profound mitotic heterogeneity. Nat Commun 11, 1-18 (2020).

25 Ott, H. W. et al. Calgranulins in cystic fluid and serum from patients with ovarian carcinomas. Cancer research 63, 7507-7514 (2003).

26 Mamoor, S. Prepronociceptin is differentially expressed in epithelial ovarian cancer. (2020).

27 Wang, H. et al. NEDD9 overexpression is associated with the progression of and an unfavorable prognosis in epithelial ovarian cancer. Human pathology 45, 401-408 (2014).

28 Zhang, Y. et al. Single-cell RNA-sequencing atlas reveals an MDK-dependent immunosuppressive environment in ErbB pathway-mutated gallbladder cancer. Journal of Hepatology (2021).

$29 \mathrm{Wu}, \mathrm{S}$. Z. et al. A single-cell and spatially resolved atlas of human breast cancers. Nature genetics 53, 1334-1347 (2021). 
30 Shih, P.-C. \& Mei, K.-C. Role of STAT3 signaling transduction pathways in cancer stem cell-associated chemoresistance. Drug Discov Today 26, 1450-1458 (2021).

31 Dunbier, A. K. et al. ESR1 is co-expressed with closely adjacent uncharacterised genes spanning a breast cancer susceptibility locus at 6q25. 1. PLoS genetics 7, e1001382 (2011).

32 Trapnell, C. et al. The dynamics and regulators of cell fate decisions are revealed by pseudotemporal ordering of single cells. Nature biotechnology 32, 381-386 (2014).

33 Zheng, A. et al. Long non-coding RNA LUCAT1/miR-5582-3p/TCF7L2 axis regulates breast cancer stemness via Wnt/ $\beta$-catenin pathway. Journal of Experimental \& Clinical Cancer Research 38, 1-14 (2019).

34 Garcia-Heredia, J. M., Lucena-Cacace, A., Verdugo-Sivianes, E. M., Pérez, M. \& Carnero, A. The cargo protein MAP17 (PDZK1IP1) regulates the cancer stem cell pool activating the Notch pathway by abducting NUMB. Clinical Cancer Research 23, 3871-3883 (2017).

35 Zhuang, Y., Li, X., Zhan, P., Pi, G. \& Wen, G. MMP11 promotes the proliferation and progression of breast cancer through stabilizing Smad2 protein. Oncology Reports $\mathbf{4 5}$, 1-1 (2021).

36 de Vega, R. G. et al. MMP-11 as a biomarker for metastatic breast cancer by immunohistochemical-assisted imaging mass spectrometry. Analytical and bioanalytical chemistry 411, 639-646 (2019).

37 Chatterjee, S. Artefacts in histopathology. Journal of oral and maxillofacial pathology: JOMFP 18, S111 (2014).

$38 \mathrm{Hu}$, J. et al. Statistical and machine learning methods for spatially resolved transcriptomics with histology. Computational and Structural Biotechnology Journal 19, 3829-3841 (2021).

39 Deng, Y. et al. Spatial-ATAC-seq: spatially resolved chromatin accessibility profiling of tissues at genome scale and cellular level. bioRxiv (2021).

40 Li, R. \& Zhou, S. Spatially resolved proteomics identify biomarkers from endometrial sentinel lymph nodes. Cell Reports Medicine 2, 100283 (2021).

41 Liu, Y. et al. High-spatial-resolution multi-omics sequencing via deterministic barcoding in tissue. Cell 183, 1665-1681. e1618 (2020).

42 Greenwald, N. F. et al. Whole-cell segmentation of tissue images with human-level performance using large-scale data annotation and deep learning. bioRxiv (2021).

43 Yi, F., Huang, J., Yang, L., Xie, Y. \& Xiao, G. Automatic extraction of cell nuclei from H\&E-stained histopathological images. Journal of Medical Imaging 4, 027502 (2017).

$44 \mathrm{Li}, \mathrm{Q}$., Han, Z. \& Wu, X.-M. in Thirty-Second AAAI conference on artificial intelligence.

45 Bahdanau, D., Cho, K. \& Bengio, Y. Neural machine translation by jointly learning to align and translate. arXiv preprint arXiv:1409.0473 (2014).

46 GENOMICS, X. Invasive Ductal Carcinoma Stained With Fluorescent CD3 Antibody, $<$ https://www.10xgenomics.com/resources/datasets/invasive-ductal-carcinomastained-with-fluorescent-cd-3-antibody-1-standard-1-2-0> (2021).

47 GENOMICS, X. Human Ovarian Cancer: Whole Transcriptome Analysis. Stains: DAPI, Anti-PanCK, Anti-CD45, $<$ https://www.10xgenomics.com/resources/datasets/human-ovarian-cancer-wholetranscriptome-analysis-stains-dapi-anti-pan-ck-anti-cd-45-1-standard-1-2-0> (2021).

48 Goldman, M. J. et al. Visualizing and interpreting cancer genomics data via the Xena platform. Nature biotechnology 38, 675-678 (2020). 
49 Netanely, D., Avraham, A., Ben-Baruch, A., Evron, E. \& Shamir, R. Expression and methylation patterns partition luminal-A breast tumors into distinct prognostic subgroups. Breast Cancer Research 18, 1-16 (2016).

50 Stuart, T. et al. Comprehensive Integration of Single-Cell Data. Cell 177, 1888-+, doi:10.1016/j.cell.2019.05.031 (2019).

51 Russell, B. C., Torralba, A., Murphy, K. P. \& Freeman, W. T. LabelMe: a database and web-based tool for image annotation. International journal of computer vision 77, 157 173 (2008).

52 Bradski, G. \& Kaehler, A. Learning OpenCV: Computer vision with the OpenCV library. (" O'Reilly Media, Inc.", 2008).

53 Yeung, K. Y. \& Ruzzo, W. L. Details of the adjusted rand index and clustering algorithms, supplement to the paper an empirical study on principal component analysis for clustering gene expression data. (2001).

54 McDaid, A. F., Greene, D. \& Hurley, N. Normalized mutual information to evaluate overlapping community finding algorithms. arXiv preprint arXiv:1110.2515 (2011).

\section{Author contributions}

L.C. and C.Z. conceived and supervised the study. C.Z. designed and implemented the model, performed all the experiments, and wrote the manuscript with feedback from all authors. Y.Z., C.C., J.F., and M. J. analyzed and interpretated the data. L.C. revised the manuscript. The authors read and approved the final manuscript.

\section{Competing interests}

The authors declare no competing interests. 


\section{Figure legends}

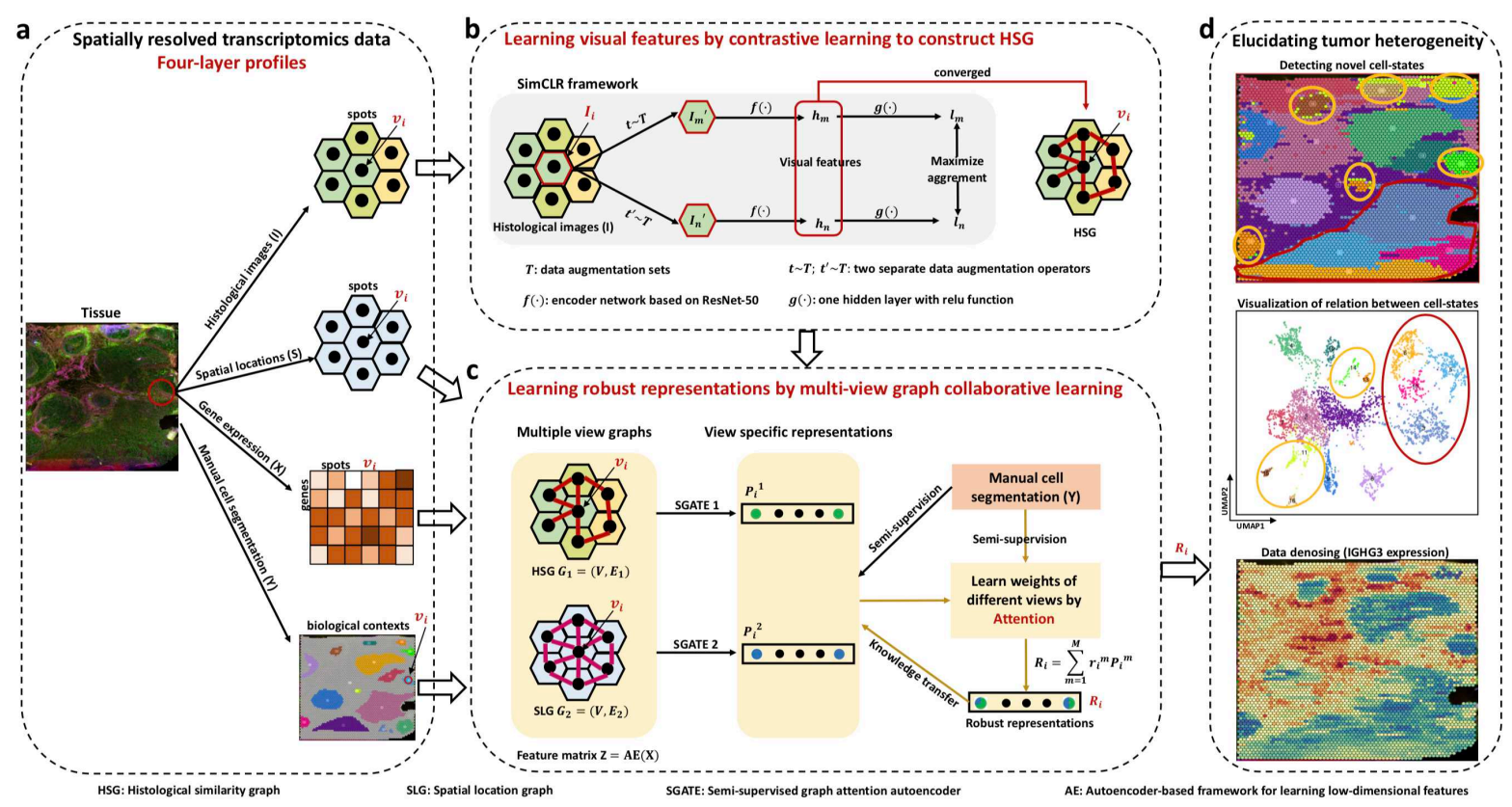

Fig.1. Overview of stMVC model. a Given each SRT data with four-layer profiles: histological images $(I)$, spatial locations $(S)$, gene expression $(X)$, and manual cell segmentation $(Y)$ as the input, stMVC integrated them to disentangle tissue heterogeneity, particularly for the tumor. $\mathbf{b}$ stMVC adopted SimCLR model with feature extraction framework from ResNet-50 to efficiently learn visual features $\left(h_{i}\right)$ for each spot $\left(v_{i}\right)$ by maximizing agreement between differently augmented views of the same spot image $\left(I_{i}\right)$ via a contrastive loss in the latent space $\left(l_{i}\right)$, and then constructed HSG by the learned visual features $h_{i}$. c stMVC model adopting a SGATE learned view-specific representations $\left(P_{i}{ }^{1}\right.$ and $\left.P_{i}{ }^{2}\right)$ for each of two graphs including HSG and SLG, as well as the latent feature from gene expression data by the autoencoder-based framework as a feature matrix, where a SGATE for each view was trained under weak supervision of the cell segmentation to capture its efficient low-dimensional manifold structure, and simultaneously integrated two-view graphs for robust representations $\left(R_{i}\right)$ by learning weights of different views via attention mechanism. $\mathbf{d}$ Robust representations $R_{i}$ can be used for elucidating tumor heterogeneity: detecting spatial domains, visualizing the relationship distance between different domains, and further denoising data. 


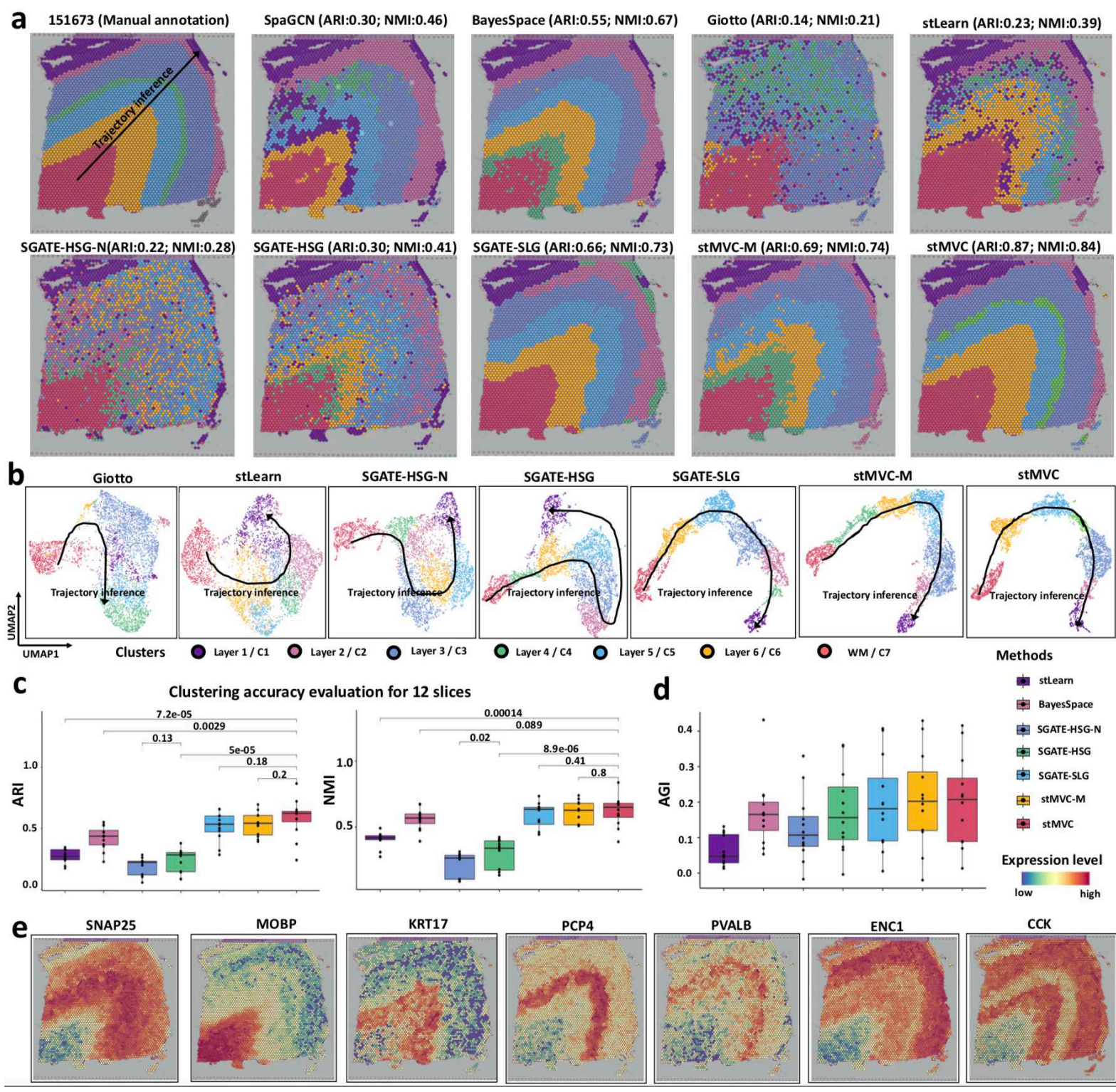

Fig.2. stMVC is able to detect spatial domains, visualize the relationship distance between different domains, and denoise data on the DLPFC dataset. a Spatial domains annotated by the previous study and detected by SpaGCN, BayesSpace, Giotto, stLearn, all SGATE-based single-view models, stMVC-M, and stMVC. b Scatter plot of the two UMAPs extracted from the latent features by Giotto, stLearn, all SGATE-based single-view models, stMVC-M, and stMVC. c Boxplot of clustering score including ARI and NMI for 12 slices by Giotto, stLearn, all SGATE-based single-view models, stMVC-M, and stMVC. d Boxplot of the AGI score of gene expression data denoised by stLearn, BayesSpace, all SGATE-based single-view models, stMVC-M, and stMVC on slice 151673. The higher AGI score, the better the denoised data. e Spatial expression of layer-specific genes: SNAP25, MOBP, KRT17, PCP4, PVALB, ENC1, and CCK for slice 151673 data denoised by stMVC. 


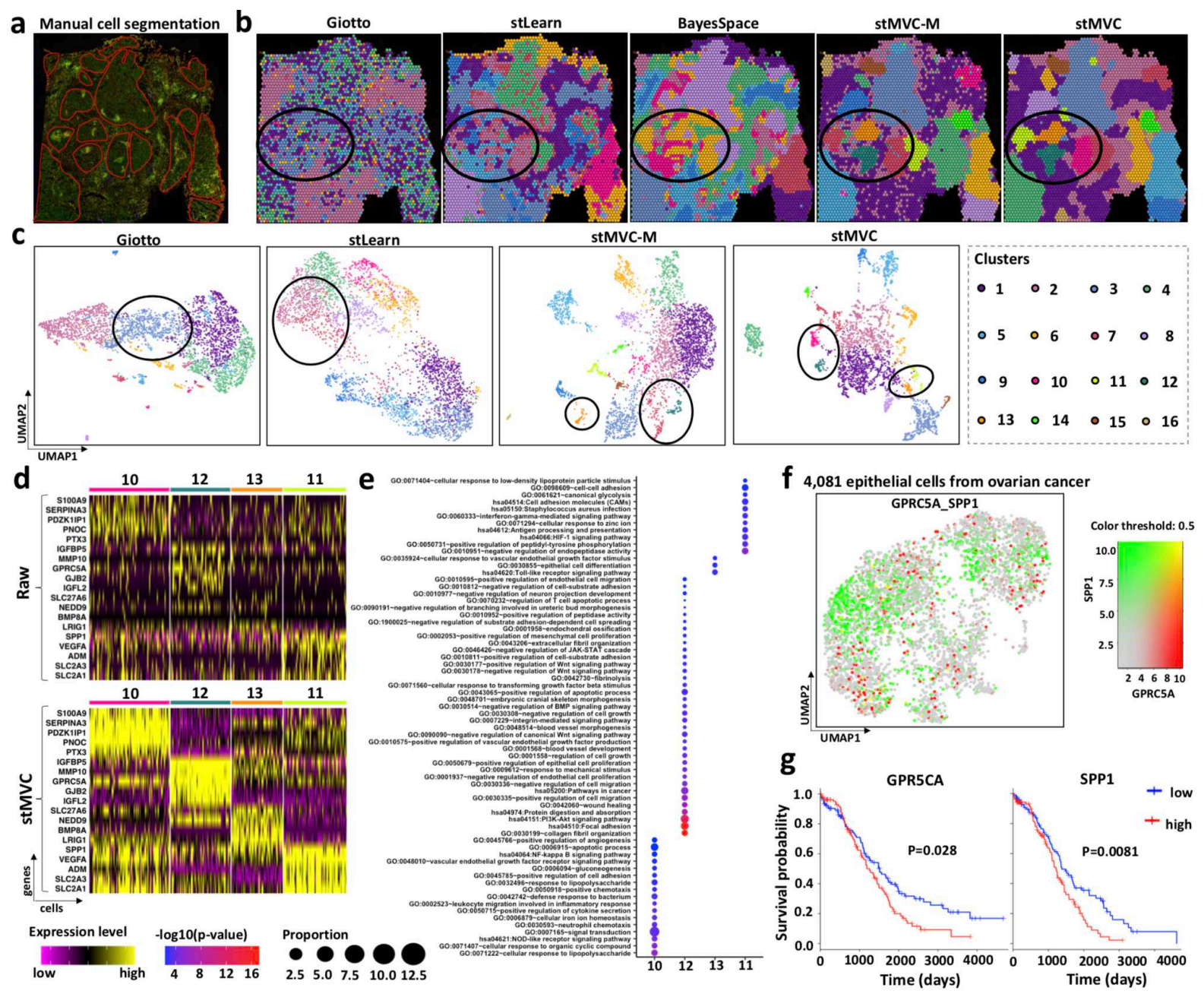

Fig.3. stMVC is able to detect different cell states distributed in the different spatial domains on the OEAD sample (ovarian cancer, $n=3,493$ spots). a Immunofluorescent staining of the tissue section and manual cell segmentation with 17 regions enriched in the tumor. The intensity of DAPI, cytokeratin and CD45 is shown in blue, green, and yellow, respectively. 17 regions are outlined with red color. b Spatial clustering by Giotto, stLearn, BayesSapce, stMVC-M, and stMVC model. c UMAP visualization of the latent features by Giotto, stLearn, stMVC-M, and stMVC model. d Heatmap of the gene expression of SVGs identified from four clusters of stMVC, as well as the raw data as a comparison. We show the top five SVGs with respect to the average log-fold-change in each domain. e Gene function enrichment analysis of SVGs in each of four clusters by DAVID. Dot size and color indicate the percentage and $p-$ value of each function in each cluster, respectively. f UMAP plot of the expression levels of GPRC5A and SPP1 in the scRNA-seq data of 4,081 epithelial cells from one ovarian cancer. g Total survival rate of patients with the high or low expression level of GPRC5A ( $p=0.028)$ and SPP1 $(p=0.0081)$ in the gene expression data of ovarian cancer from TCGA database. The logrank test was used for the survival analysis. These ovarian cancers were classified into two 
groups based on their expression (high $>75 \%$ of value, low $<25 \%$ of value) for comparison of survival.

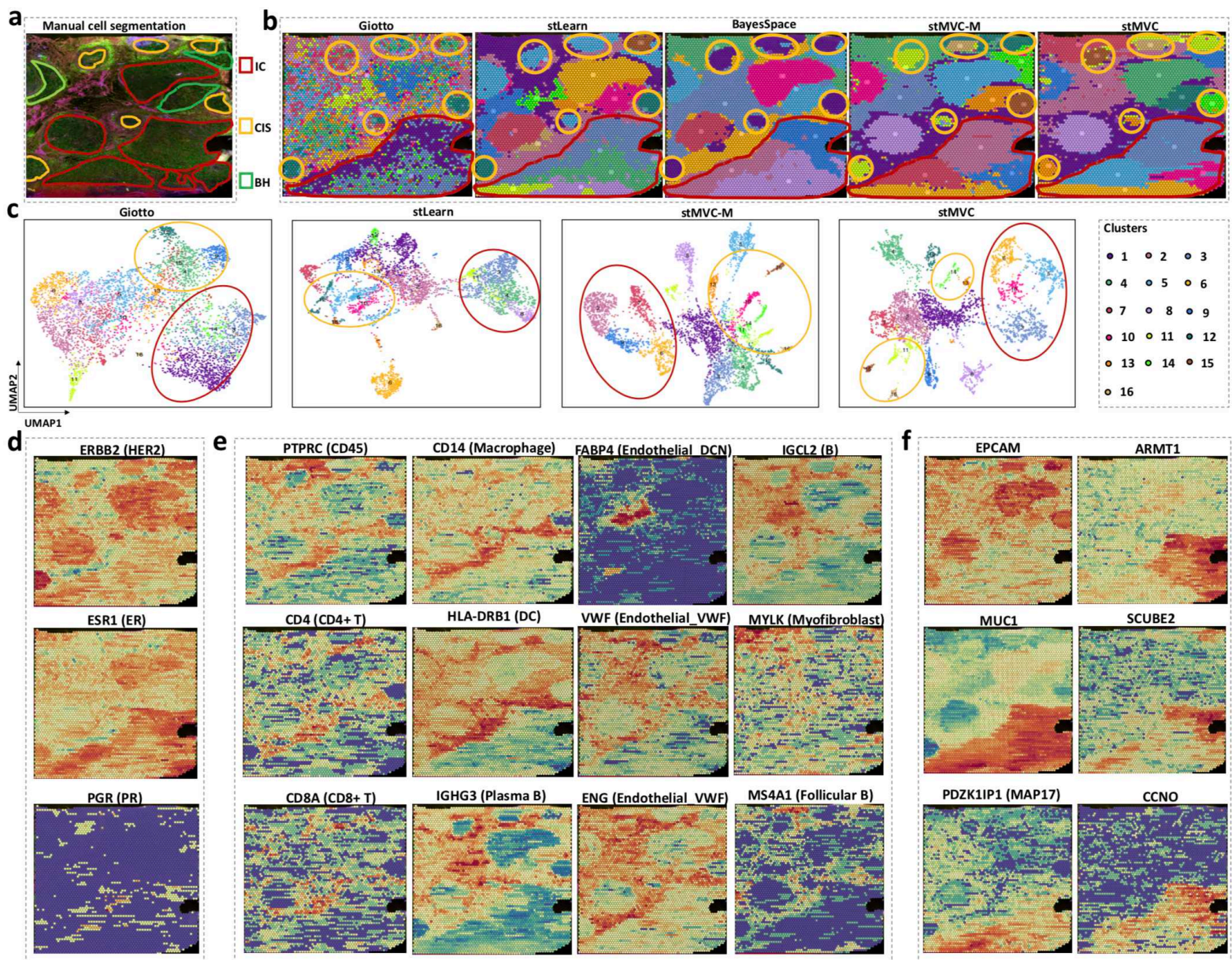

Fig.4. stMVC enables disentangling tumor heterogeneity in IDC sample (breast cancer, $n=$ 4,727 spots). a Immunofluorescent staining of the tissue section and manual cell segmentation with 15 regions enriched in the tumor. The intensity of DAPI, fiducial frame, and anti-CD3 is shown in green, blue, and yellow, respectively. The annotated regions are outlined based on their histological annotations. Specifically, IC, CIS, and BH regions are outlined with red, yellow, and green color, respectively. b Spatial clustering by Giotto, stLearn, BayesSapce, stMVC-M, and stMVC model. c UMAP visualization of the latent features by Giotto, stLearn, stMVC-M, and stMVC model. d Spatial expression of genes for HER2 (ERBB2), ER (ESR1), and PR (PGR) for data denoised by stMVC. e Spatial expression of marker genes of non-tumor cells, such as PTPRC (CD45), CD4, CD8A, CD14, HLA-DRB1, IGHG3, FABP4, VWF, ENG, IGCL2, MYLK, and MS4A1 for data from stMVC. f Spatial expression of makers of tumor progression genes: EPCAM, MUC1, MAP17, ARMT1, SCUBE2, and CCNO for data from stMVC. 

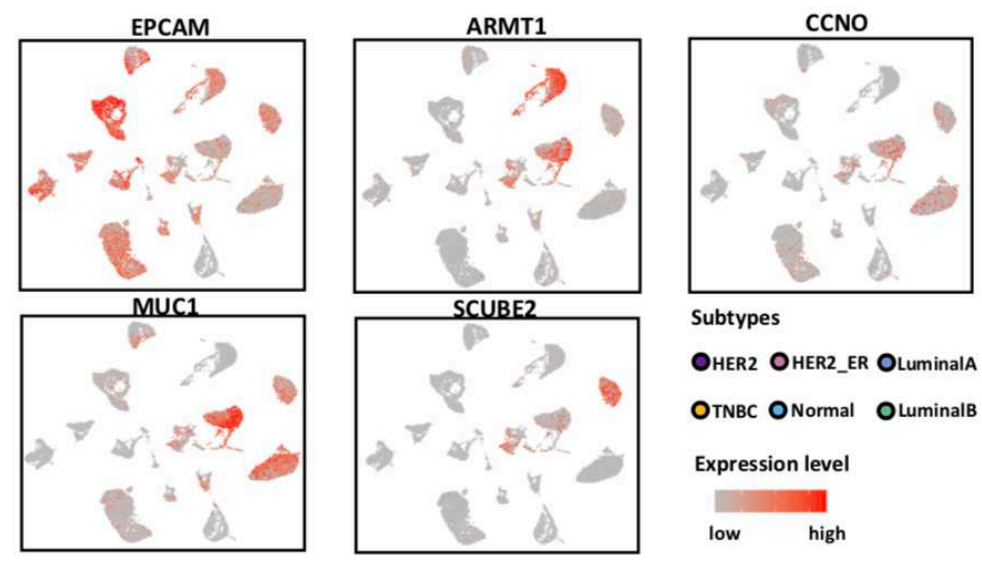

Subtypes

OHer2 OHer2_er OLuminala

OTNBC ONormal OLuminalB

Expression level

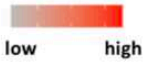

b \begin{tabular}{l|ll}
\hline UMAP1 & 6 & 10 \\
\hline
\end{tabular}
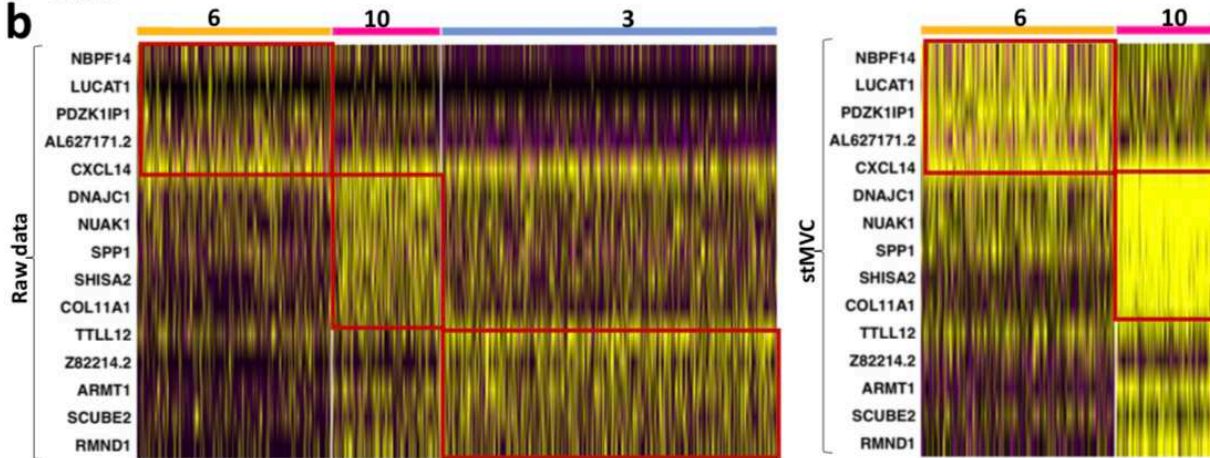

3

C

2,352 epithelial cells from CID4067 patient

Clustering

PDZK1IP1
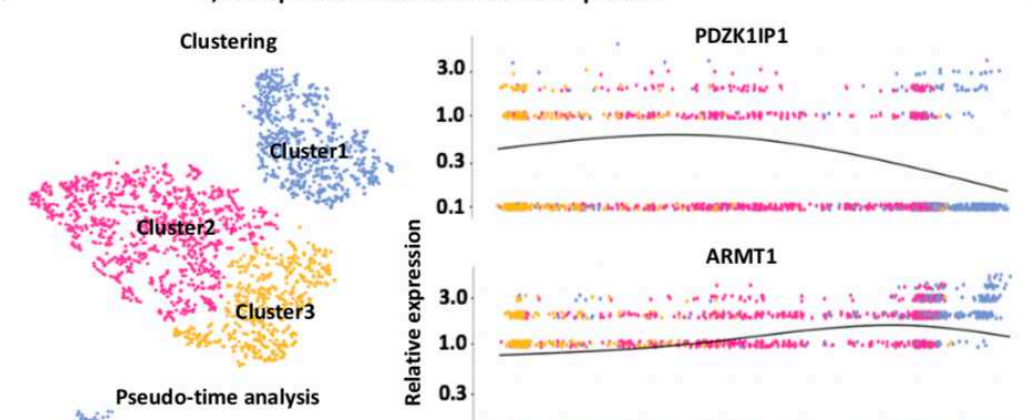

d
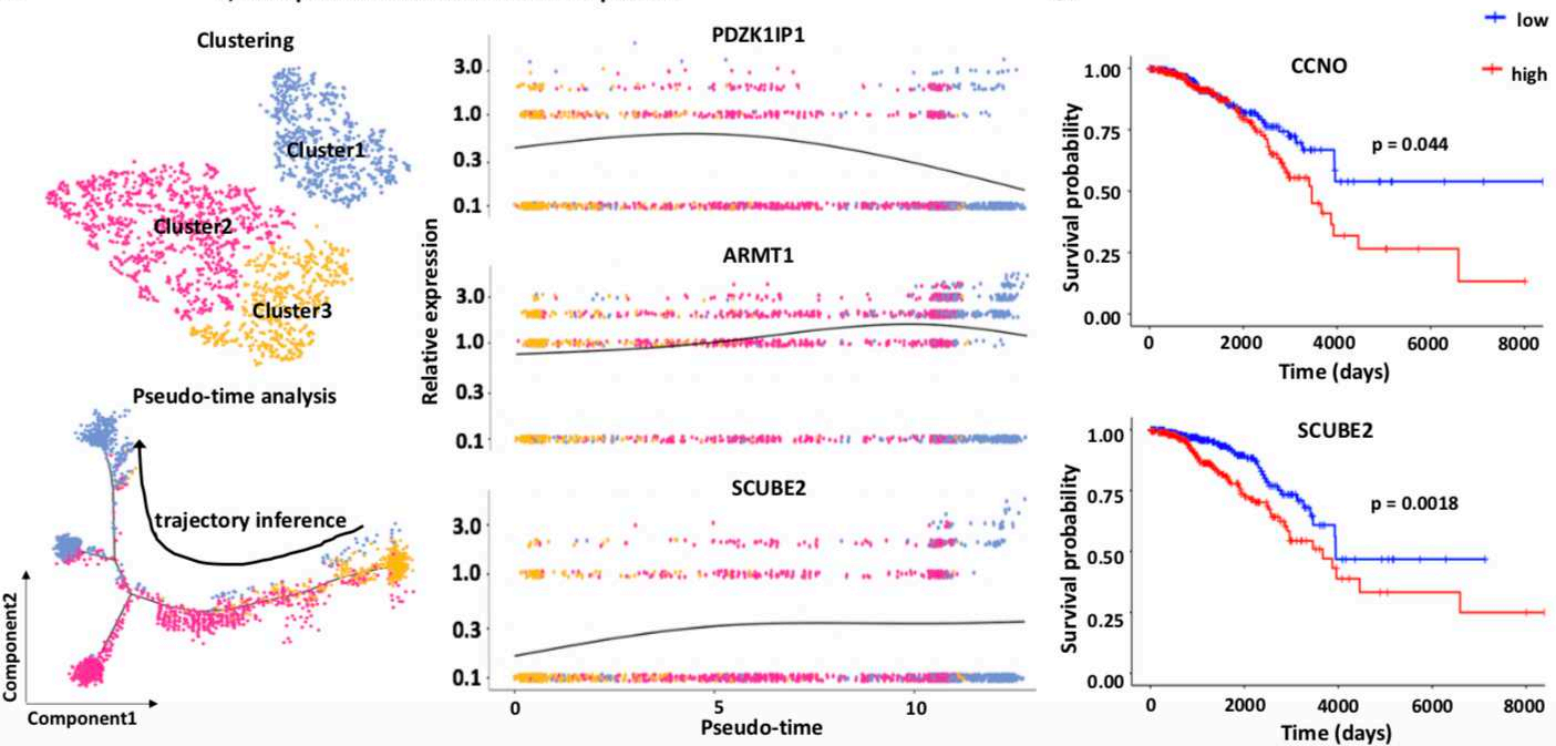

Fig.5. stMVC enables identifying novel tumor-related cell-states and their transition cell-states from IC region in IDC sample. a UMAP visualization of 24,489 epithelial cells from 20 breast cancer patients, as well as the expression levels of EPCAM, MUC1, ARMT1, SCUBE2, and CCNO. Each color indicates one subtype of breast cancer determined based on the status of ER, PR, and HER2. b Heatmap of the gene expression of SVGs identified from four stMVC clusters, as well as the raw data as a comparison. We show the top five SVGs with respect to average log-fold-change in each domain. c Visualization of the clustering and trajectory 
inference from 2,352 epithelial cells of CID4067 (a luminal B patient), and pseudo-timedependent changes in expression levels of ARMT1, SCUBE2, and CCNO. d Total survival rate of patients with the high or low expression level of CCNO $(p=0.044)$ and SCUBE2 $(\mathrm{p}=0.0018)$ in the RNA-seq data of breast cancer from TCGA database. The logrank test was used for the survival analysis. These breast cancer patients were classified into two groups based on their expression (high $>$ mean value, low $<$ mean value) for comparison of survival. 


\section{Supplementary Files}

This is a list of supplementary files associated with this preprint. Click to download.

- Supplementarymaterial.pdf 